\title{
The atmospheric transparency of Telescope Array observation site by the CLF
}

\author{
Takayuki Tomida, ${ }^{a, *}$ Tomoyuki Nakamura, ${ }^{a, *}$ Katsuya Yamazaki, ${ }^{b}$ John Matthews ${ }^{c}$ \\ and for the Telescope Array collaboration on behalf of the Telescope Array \\ Collaboration \\ (a complete list of authors can be found at the end of the proceedings) \\ ${ }^{a}$ Academic Assembly School of Science and Technology Institute of Engineering, Shinshu University, \\ Nagano, Nagano, Japan \\ ${ }^{b}$ Engineering Science LaboratoryCollege of EngineeringChubu University, \\ Kasugai-shi, Aichi, Japan \\ ${ }^{c}$ Department of Physics and High Energy Astrophysics Institute, The University of Utah, \\ Salt Lake City, Utah, USA \\ E-mail: tomida@cs.shinshu-u.ac.jp
}

The Telescope Array (TA) experiment continues to observe Ultra High Energy Cosmic Rays (UHECRs) both with its original TA detectors as well as with the new TAx4 expansion detectors. These observations employ Fluorescence Detectors (FDs) to capture the air shower induced by the primary UHECRs. The FD observes fluorescence light emitted from atmospheric nitrogen molecules excited by air shower particles. The observation of the FD extends over tens of kilometers, and the fluorescence light is attenuated by scattering from atmospheric molecules and aerosols during the propagation process. Seasonal dependence was found when assessing the attenuation of fluorescence by aerosols. We also captured the weather characteristics. We report on the effect of aerosols on the atmospheric transparency of the TA sites.

$37^{\text {th }}$ International Cosmic Ray Conference (ICRC 2021)

July 12th - 23rd, 2021

Online - Berlin, Germany

\footnotetext{
${ }^{*}$ Presenter
} 


\section{Introduction}

The Telescope Array experiment keeps observing Ultra High Energy Cosmic Ray(UHECR) from the beginning of 2008 to the present. The TA uses two air shower detection techniques of fluorescence detection technique and particle surface detection technique to observe UHECRs[1][2]. A layout of TA experiment site is shown in Figure 1. The TA consists of 507 surface detectors (SDs) and 3 fluorescence detector(FD) stations. Each FD station called as "Black Rock Mesa"(BR), "Long Ridge"(LR) and "Middle Drum"(MD) have been installed surrounding SD array.

Fluorescence light witch is generated by the air shower. FD observes cosmic rays indirectly by detecting these fluorescence light. Atmospheric monitoring is necessary for observing UHECRs using the air fluorescence technique because the fluorescence light is scattered by the atmosphere in their path of transmission to the FD. Mainly, two types of scattering are considered the atmospheric molecules and the aerosol. Rayleigh scattering that the atmospheric molecules causes can estimate from information of the temperature and the atmospheric pressure obtained from the GDAS[3]. Scattering caused by an aerosol has significant effect on energy of cosmic rays because the shape, the amount and the composition of the aerosol are varied by the atmospheric and ground situation. Moreover, they changes time by time.

In the TA experiment, we employ a variety of measurements for atmospheric monitoring, using laser systems. This laser system is located at the center of three FD stations, and the light scattered by the atmosphere is observed by each fluorescence detector station. This system is called CLF(Central Laser Facility), that overview is shown in Fig. 2[4]. In the last ICRC, We have been reporting on the Vertical Aerosol Optical Depth (VAOD) as the atmospheric transparency obtained from CLF observations. Since the VAOD from the previous analysis did not consider the annual fluctuation of atmospheric molecules, the influence of the annual fluctuation of atmospheric molecules was removed in this work. In this the paper, we report about system, analysis and obtained

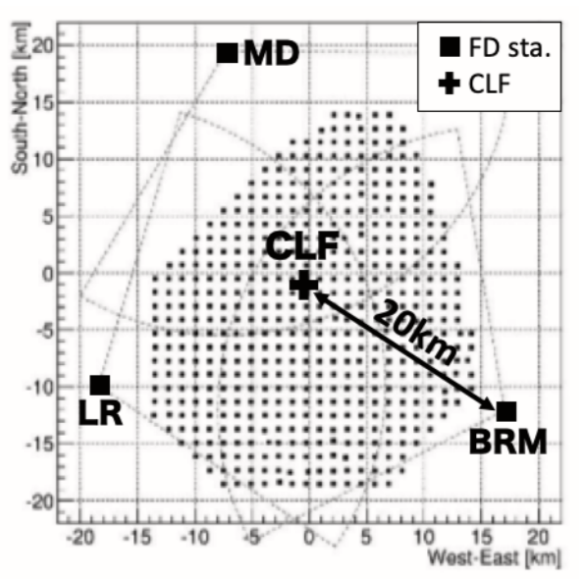

Figure 1: A layout of the TA experiment site. Black small squares are SDs, and three FD sta- ${ }^{\mathrm{p}}$ tions are shown by BR, LR, and MD with large square. CLF is located at the center of them.

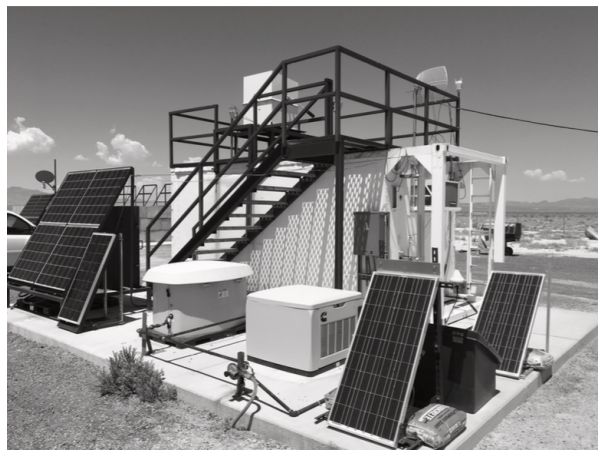

Figure 2: The CLF system. The piture is appearance of the CLF container. 
atmospheric transparency of the CLF.

\section{Analysis Method}

In order to analyze the laterally scattered light of the vertical laser by the CLF and obtain atmospheric attenuation only by aerosol, we compare it with the state where the aerosol is extremely low, that is, the state where atmospheric scattering by atmospheric molecules is dominant. We have selected data similar to ideal observations in which atmospheric molecules have a dominant effect on all atmospheric scattering. The details will be described later, but it was decided that the observation with the largest amount of light received from the altitude where the weather is sunny and the aerosol is thin is similar to the ideal observation situation.

\subsection{Vertical Aerosol Optical Depth}

Using the CLF system, we can obtain Vertical Aerosol Optical Depth (VAOD) by considering Rayleigh scattering and the detection efficiency of FD. The VAOD $\tau_{\mathrm{AS}}$ is a value equal with the integration value of $\alpha$ to $\mathrm{H} \mathrm{km}$ from $0 \mathrm{~km}(2) . \alpha$ is extinction coefficient which is the reciprocal of the attenuation length as a function of height $h$.

$$
\tau_{\mathrm{AS}}(\mathrm{H})=\int_{0}^{\mathrm{H}} \alpha_{\mathrm{AS}}(h) d h .
$$

The VAOD obtained by CLF reflects accurately the total amount of aerosols because the photon passes at a low altitude during the propagation period from the air shower to FD(see Figure 3). Thus, we can calibration factor of the atmosphere every 30 minutes within the FD observation time.

\subsection{Solution policy}

Overview of the laser path in the analysis is shown in Figure 3. The number of photons $N p_{0}$ at laser burst is attenuated by atmospherical molecules and aerosol. The attenuation factor $T(\mathrm{H})$ for photons which vertically propagate in the atmosphere at height $\mathrm{H}$ is given by Equation 3.1

$$
T_{\mathrm{AS}}(\mathrm{H})=\exp \left[-\tau_{\mathrm{AS}}(\mathrm{H})\right]
$$

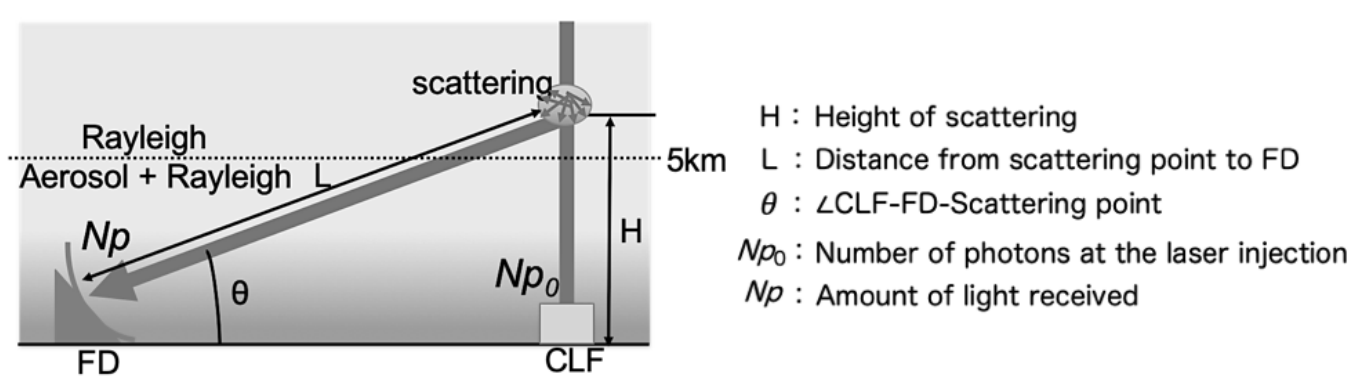

Figure 3: Overview of the laser path in the analysis. 
Suffix "AS" means for "Aerosol", and "Ray" means for "Rayleigh" scattering. After attenuated from the ground to height $\mathrm{H}$ and scattered, the amount of light heading to the FD can be calculated from the scattering coefficient $S$. $S$ is a coefficient including scattering cross-section and angle. The received light amount of FD is shown in Equation 3.

$$
N p(\mathrm{H})=N p_{0} T_{\text {Ray }}(\mathrm{H}) T_{\mathrm{AS}}(\mathrm{H})\left(S_{\text {Ray }}+S_{\mathrm{AS}}\right) T_{\mathrm{Ray}}(\mathrm{L}) T_{\mathrm{AS}}(\mathrm{L}) .
$$

Assuming the amount of light received $N p^{\prime}$ in the atmosphere without aerosol at the clear night, there is no scattering by aerosol. Therefore, The number of photons $N p_{0}^{\prime}$ at laser burst is scattered by only atmospherical molecules at the clear night. $N p^{\prime}$ at the clear night is shown in Equation 4 where it assumed $T_{\mathrm{AS}}(H)=T_{\mathrm{AS}}(L)=1$ and $S_{\mathrm{AS}}=0$.

$$
N p^{\prime}(\mathrm{H})=N p_{0}^{\prime} T_{\text {Ray }}^{\prime}(\mathrm{H}) S_{\text {Ray }}^{\prime} T_{\text {Ray }}^{\prime}(\mathrm{L}) .
$$

With this taken into consideration, we analyze assuming the following three conditions.

- Use scattered at higher than $5 \mathrm{~km}$ height data in measured waveform (atmospherical molecule dominant)

- Assume the atmospheric parameters are constant in horizontal direction

- We assumed that the effects of annual fluctuations in atmospheric molecules would be negligible. That is shown as following:

$$
T_{\text {Ray }}(H)=T_{\text {Ray }}^{\prime}(H), T_{\text {Ray }}(L)=T_{\text {Ray }}^{\prime}(L) \text { and } S_{\text {Ray }}=S_{\text {Ray }}^{\prime} .
$$

First, Mie-LIDAR by TA experiment has found that aerosols do not exist at a height of $5 \mathrm{~km}$ or more from the ground at the TA site[4]. In this analysis, it is analyzed CLF waveforms of higher than $5 \mathrm{~km}$ of height data because scattering by aerosol is negligible at that height. Therefore, we can assume $S_{\mathrm{AS}}=0$. Second, assume that the lateral aerosol distribution for each altitude at the TA experiment site is constant. Thus, attenuation factor $\mathrm{T}(\mathrm{L})$ is calculated by $T(\mathrm{~L})=T(\mathrm{H}) / \sin \theta$. $\theta$ is determined from the light receiving timing recorded by PMTs of FD. Based on these two conditions, VAOD $\tau_{\text {AS }}$ is obtained by comparing $N p$ with $N p^{\prime}$ at the clear night(Equation 5).

$$
\frac{N p(\mathrm{H})}{N p^{\prime}(\mathrm{H})}=\frac{N p_{0}}{N p_{0}^{\prime}} \exp \left[-\tau_{\mathrm{AS}}(\mathrm{H}) \frac{\sin \theta+1}{\sin \theta}\right]
$$

Solving this equation as a solution for $\tau_{\mathrm{AS}}$ gives:

$$
\tau_{\mathrm{AS}}(\mathrm{H})=-\frac{\sin \theta+1}{\sin \theta} \ln \left[\frac{N p_{0}^{\prime}}{N p_{0}} \frac{N p(\mathrm{H})}{N p^{\prime}(\mathrm{H})}\right] .
$$

This solution assumes that atmospheric molecules do not fluctuate over the years. However, the optical depth of atmospheric molecules from the ground to $5 \mathrm{~km}$ above the ground at ELKO near the TA experimental site varies by $8 \%$ annually, and the extinction coefficient $\alpha_{\text {Ray }}(5 \mathrm{~km})$ at a surface altitude of $5 \mathrm{~km}$ fluctuates by $3 \%$. Since we thought that the effects of scattering due to annual fluctuations of atmospheric molecules should be fully considered, we sought a solution that took into account the effects of atmospheric molecules. Therefore, we newly added the following conditions: $T_{\text {Ray }}(H) \neq T_{\text {Ray }}^{\prime}(H), T_{\text {Ray }}(L) \neq T_{\text {Ray }}^{\prime}(L)$ and $S_{\text {Ray }}=S_{\text {Ray }}^{\prime}$. 


$$
\begin{aligned}
\frac{N p(\mathrm{H})}{N p^{\prime}(\mathrm{H})}= & \frac{N p_{0}}{N p_{0}^{\prime}} \exp \left[-\tau_{\mathrm{AS}}(\mathrm{H}) \frac{\sin \theta+1}{\sin \theta}\right] \\
& \quad \exp \left[-\tau_{\text {Ray }}(\mathrm{H})+\tau_{\text {Ray }}^{\prime}(\mathrm{H})\right] \exp \left[\frac{-\tau_{\text {Ray }}(\mathrm{H})+\tau_{\text {Ray }}^{\prime}(\mathrm{H})}{\sin \theta}\right] \exp \left[\left(-\alpha_{\text {Ray }}+\alpha_{\text {Ray }}^{\prime}\right) \Delta \mathrm{H}\right] .
\end{aligned}
$$

As with the equation 6 , the solution for $\tau_{\mathrm{AS}}(\mathrm{H})$ is as follows.

$$
\tau_{\mathrm{AS}}(\mathrm{H})=-\frac{\sin \theta+1}{\sin \theta} \ln \left[\frac{N p_{0}^{\prime}}{N p_{0}} \frac{N p(\mathrm{H})}{N p^{\prime}(\mathrm{H})}\right]+\left(\left(\tau_{\text {Ray }}^{\prime}(\mathrm{H})-\tau_{\text {Ray }}(\mathrm{H})\right)+\frac{\sin \theta+1}{\sin \theta}\left(\alpha_{\text {Ray }}^{\prime}-\alpha_{\text {Ray }}^{\prime}\right) \Delta \mathrm{H}\right)
$$

\section{Result and Discussion}

We calculated the VAOD $\tau_{\mathrm{AS}}(5 \mathrm{~km})$ at the BR station using CLF by 1853 events from January 2012 to September 2016. We divided the period by the maintenance of the equipment etc., and secured three periods and analyzed by each observation period. Figure 4 shows the monthly transition of the median VAOD for three years. It shows the results of previous and this work. The effect in summer was remarkable, and a 5 10\% increase in VAOD was confirmed. The median and distribution $(1 \sigma)$ of VAOD in three years by each of previous and this work are $0.044_{-0.017}^{+0.025}$ and $0.045_{-0.018}^{+0.026}$. This statistic is evaluated with a distribution with a VAOD that is less than 0.02 . This distribution is shown in Fig. 5.

The obtained VAOD was a change within the distribution on average over 3 years. However, the effect was confirmed depending on the season. In the future, the seasonal dependence of aerosols should be considered in the FD analysis of TA experiments.

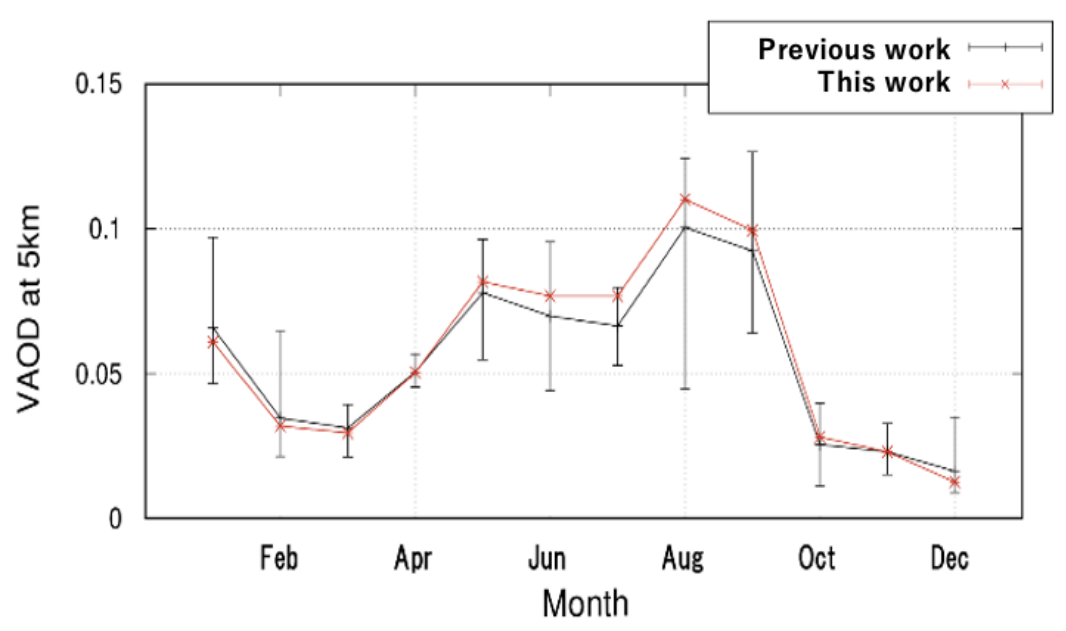

Figure 4: Median of the VAOD for monthly transition. 


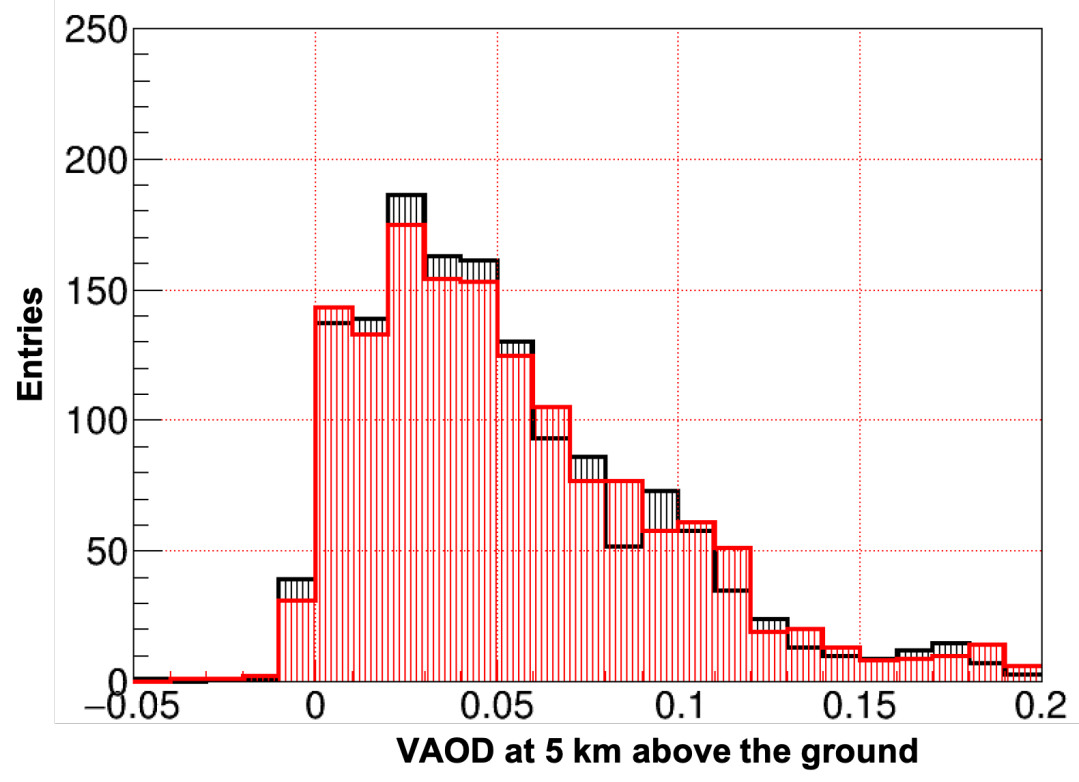

Figure 5: Distribution of VAOD for 3 years.

\section{Acknowledgements}

The Telescope Array experiment is supported by the Japan Society for the Promotion of Science (JSPS) through Grants-in-Aid for Priority Area 431, for Specially Promoted Research JP21000002, for Scientific Research (S) JP19104006, for Specially Promoted Research JP15H05693, for Scientific Research (S) JP15H05741, for Science Research (A) JP18H03705, for Young Scien- tists (A) JP17K14280 and for Young Scientists (B). JPH26707011; by the joint research program of the Institute for Cosmic Ray Research (ICRR), The University of Tokyo; by the U.S. National Science Foundation awards PHY-0601915, PHY-1404495, PHY-1404502, and PHY-1607727; by the National Research Foundation of Korea (2016R1A2B4014967, 2016R1A5A1013277, 2017K1A4A3015188, 2017R1A2A1A05071429); by the Russian Academy of Sciences, RFBR grant 16-02-00962a (INR), IISN project No. 4.4502.13, and Belgian Science Policy under IUAP VII/37 (ULB). The foundations of Dr. Ezekiel R. and Edna Wattis Dumke, Willard L. Eccles, and George S. and Dolores Doré Eccles all helped with generous donations. The State of Utah sup- ported the project through its Economic Development Board, and the University of Utah through the Office of the Vice President for Research. The experimental site became available through the cooperation of the Utah School and Institutional Trust Lands Administration (SITLA), U.S. Bureau of Land Management (BLM), and the U.S. Air Force. We appreciate the assistance of the State of Utah and Fillmore offices of the BLM in crafting the Plan of Development for the site. Patrick Shea assisted the collaboration with valuable advice on a variety of topics. The people and the officials of Millard County, Utah have been a source of steadfast and warm support for our work which we greatly appreciate. We are indebted to the Millard County Road Department for their efforts to maintain and clear the roads which get us to our sites. We gratefully acknowledge the contribution from the technical staffs of our home institutions. An allocation of computer time from the Center for High Performance 
Computing at the University of Utah is gratefully acknowledged.

\section{References}

[1] H.Tokuno et al. ,NIM A, 676, 54-65 (2012)

[2] T.Abu-Zayyad et al. ,NIM A, 689, 87-97 (2012)

[3] https://www.ncdc.noaa.gov/data-access/model-data/model-datasets/global-data-assimilationsystem-gdas

[4] T.Tomida et al. ,EPJ Web of Conferences 53, 10003 (2013) 


\section{Full Authors List: Telescope Array Collaboration}

R.U. Abbasi ${ }^{1,2}$, T. Abu-Zayyad ${ }^{1,2}$, M. Allen ${ }^{2}$, Y. Arai $^{3}$, R. Arimura ${ }^{3}$, E. Barcikowski' ${ }^{2}$, J.W. Belz ${ }^{2}$, D.R. Bergman ${ }^{2}$, S.A. Blake ${ }^{2}$, I. Buckland ${ }^{2}$, R. Cady $^{2}$, B.G. Cheon ${ }^{4}$, J. Chiba ${ }^{5}$, M. Chikawa ${ }^{6}$, T. Fujii ${ }^{7}$, K. Fujisue ${ }^{6}$, K. Fujita ${ }^{3}$, R. Fujiwara ${ }^{3}$, M. Fukushima ${ }^{6}$, R. Fukushima ${ }^{3}$, G. Furlich ${ }^{2}$, R. Gonzalez ${ }^{2}$, W. Hanlon ${ }^{2}$, M. Hayashi ${ }^{8}$, N. Hayashida ${ }^{9}$, K. Hibino ${ }^{9}$, R. Higuchi ${ }^{6}$, K. Honda ${ }^{10}$, D. Ikeda ${ }^{9}$, T. Inadomi ${ }^{11}$, N. Inoue ${ }^{12}$, T. Ishii ${ }^{10}$, H. Ito ${ }^{13}$, D. Ivanov ${ }^{2}$, H. Iwakura ${ }^{11}$, A. Iwasaki ${ }^{3}$, H.M. Jeong ${ }^{14}$, S. Jeong ${ }^{14}$, C.C.H. Jui ${ }^{2}$, K. Kadota ${ }^{15}$, F. Kakimoto ${ }^{9}$, O. Kalashev ${ }^{16}$, K. Kasahara ${ }^{17}$, S. Kasami ${ }^{18}$, H. Kawai ${ }^{19}$, S. Kawakami ${ }^{3}$, S. Kawana ${ }^{12}$, K. Kawata ${ }^{6}$, I. Kharuk ${ }^{16}$, E. Kido ${ }^{13}$, H.B. Kim ${ }^{4}$, J.H. Kim ${ }^{2}$, J.H. Kim ${ }^{2}$, M.H. Kim ${ }^{14}$, S.W. Kim ${ }^{14}$, Y. Kimura ${ }^{3}$, S. Kishigami ${ }^{3}$, Y. Kubota ${ }^{11}$, S. Kurisu ${ }^{11}$, V. Kuzmin ${ }^{16}$, M. Kuznetsov ${ }^{16,20}$, Y.J. Kwon ${ }^{21}$, K.H. Lee ${ }^{14}$, B. Lubsandorzhiev ${ }^{16}$, J.P. Lundquist ${ }^{2,22}$, K. Machida ${ }^{10}$, H. Matsumiya ${ }^{3}$, T. Matsuyama ${ }^{3}$, J.N. Matthews ${ }^{2}$, R. Mayta ${ }^{3}$, M. Minamino ${ }^{3}$, K. Mukai ${ }^{10}$, I. Myers ${ }^{2}$, S. Nagataki ${ }^{13}$, K. Nakai $^{3}$, R. Nakamura ${ }^{11}$, T. Nakamura ${ }^{23}$, T. Nakamura ${ }^{11}$, Y. Nakamura ${ }^{11}$, A. Nakazawa ${ }^{11}$, E. Nishio ${ }^{18}$, T. Nonaka ${ }^{6}$, H. Oda $^{3}$, S. Ogio $^{3,24}$, M. Ohnishi ${ }^{6}$, H. Ohoka ${ }^{6}$, Y. Oku ${ }^{18}$, T. Okuda ${ }^{25}$, Y. Omura ${ }^{3}$, M. Ono ${ }^{13}$, R. Onogi ${ }^{3}$, A. Oshima ${ }^{3}$, S. Ozawa ${ }^{26}$, I.H. Park ${ }^{14}$, M. Potts ${ }^{2}$, M.S. Pshirkov ${ }^{16,27}$, J. Remington ${ }^{2}$, D.C. Rodriguez ${ }^{2}$, G.I. Rubtsov ${ }^{16}$, D. Ryu ${ }^{28}$, H. Sagawa ${ }^{6}$, R. Sahara ${ }^{3}$, Y. Saito ${ }^{11}$, N. Sakaki ${ }^{6}$, T. Sako ${ }^{6}$, N. Sakurai ${ }^{3}$, K. Sano ${ }^{11}$, K. Sato ${ }^{3}$, T. Seki ${ }^{11}$, K. Sekino ${ }^{6}$, P.D. Shah ${ }^{2}$, Y. Shibasaki ${ }^{11}$, F. Shibata ${ }^{10}$, N. Shibata ${ }^{18}$, T. Shibata ${ }^{6}$, H. Shimodaira ${ }^{6}$, B.K. Shin ${ }^{28}$, H.S. Shin ${ }^{6}$, D. Shinto ${ }^{18}$, J.D. Smith ${ }^{2}$, P. Sokolsky ${ }^{2}$, N. Sone ${ }^{11}$, B.T. Stokes ${ }^{2}$, T.A. Stroman ${ }^{2}$, Y. Takagi ${ }^{3}$,

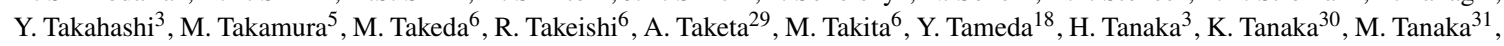
Y. Tanoue ${ }^{3}$, S.B. Thomas ${ }^{2}$, G.B. Thomson ${ }^{2}$, P. Tinyakov ${ }^{16,20}$, I. Tkachev ${ }^{16}$, H. Tokuno ${ }^{32}$, T. Tomida ${ }^{11}$, S. Troitsky ${ }^{16}$, R. Tsuda ${ }^{3}$, Y. Tsunesada ${ }^{3,24}$, Y. Uchihori ${ }^{33}$, S. Udo ${ }^{9}$, T. Uehama ${ }^{11}$, F. Urban ${ }^{34}$, T. Wong ${ }^{2}$, K. Yada ${ }^{6}$, M. Yamamoto ${ }^{11}$, K. Yamazaki ${ }^{9}$, J. Yang ${ }^{35}$, K. Yashiro ${ }^{5}$, F. Yoshida ${ }^{18}$, Y. Yoshioka ${ }^{11}$, Y. Zhezher ${ }^{6,16}$, and Z. Zundel ${ }^{2}$

${ }^{1}$ Department of Physics, Loyola University Chicago, Chicago, Illinois, USA

${ }^{2}$ High Energy Astrophysics Institute and Department of Physics and Astronomy, University of Utah, Salt Lake City, Utah, USA

${ }^{3}$ Graduate School of Science, Osaka City University, Osaka, Osaka, Japan

${ }^{4}$ Department of Physics and The Research Institute of Natural Science, Hanyang University, Seongdong-gu, Seoul, Korea

${ }^{5}$ Department of Physics, Tokyo University of Science, Noda, Chiba, Japan

${ }^{6}$ Institute for Cosmic Ray Research, University of Tokyo, Kashiwa, Chiba, Japan

${ }^{7}$ The Hakubi Center for Advanced Research and Graduate School of Science, Kyoto University, Kitashirakawa-Oiwakecho, Sakyo-ku, Kyoto, Japan

${ }^{8}$ Information Engineering Graduate School of Science and Technology, Shinshu University, Nagano, Nagano, Japan

${ }^{9}$ Faculty of Engineering, Kanagawa University, Yokohama, Kanagawa, Japan

${ }^{10}$ Interdisciplinary Graduate School of Medicine and Engineering, University of Yamanashi, Kofu, Yamanashi, Japan

${ }^{11}$ Academic Assembly School of Science and Technology Institute of Engineering, Shinshu University, Nagano, Nagano, Japan

12 The Graduate School of Science and Engineering, Saitama University, Saitama, Saitama, Japan

${ }^{13}$ Astrophysical Big Bang Laboratory, RIKEN, Wako, Saitama, Japan

${ }^{14}$ Department of Physics, SungKyunKwan University, Jang-an-gu, Suwon, Korea

${ }^{15}$ Department of Physics, Tokyo City University, Setagaya-ku, Tokyo, Japan

${ }^{16}$ Institute for Nuclear Research of the Russian Academy of Sciences, Moscow, Russia

${ }^{17}$ Faculty of Systems Engineering and Science, Shibaura Institute of Technology, Minato-ku, Tokyo, Japan

${ }^{18}$ Department of Engineering Science, Faculty of Engineering, Osaka Electro-Communication University, Neyagawa-shi, Osaka, Japan

${ }^{19}$ Department of Physics, Chiba University, Chiba, Chiba, Japan

${ }^{20}$ Service de Physique Thacuteeorique, Universitacutee Libre de Bruxelles, Brussels, Belgium

${ }^{21}$ Department of Physics, Yonsei University, Seodaemun-gu, Seoul, Korea

${ }^{22}$ Center for Astrophysics and Cosmology, University of Nova Gorica, Nova Gorica, Slovenia

${ }^{23}$ Faculty of Science, Kochi University, Kochi, Kochi, Japan

${ }^{24}$ Nambu Yoichiro Institute of Theoretical and Experimental Physics, Osaka City University, Osaka, Osaka, Japan

${ }^{25}$ Department of Physical Sciences, Ritsumeikan University, Kusatsu, Shiga, Japan

${ }^{26}$ Quantum ICT Advanced Development Center, National Institute for Information and Communications Technology, Koganei, Tokyo, Japan

27 Sternberg Astronomical Institute, Moscow M.V. Lomonosov State University, Moscow, Russia

${ }_{28}$ Department of Physics, School of Natural Sciences, Ulsan National Institute of Science and Technology, UNIST-gil, Ulsan, Korea

${ }^{29}$ Earthquake Research Institute, University of Tokyo, Bunkyo-ku, Tokyo, Japan

${ }^{30}$ Graduate School of Information Sciences, Hiroshima City University, Hiroshima, Hiroshima, Japan

${ }^{31}$ Institute of Particle and Nuclear Studies, KEK, Tsukuba, Ibaraki, Japan

32 Graduate School of Science and Engineering, Tokyo Institute of Technology, Meguro, Tokyo, Japan

33 Department of Research Planning and Promotion, Quantum Medical Science Directorate, National Institutes for Quantum and Radiological Science and Technology, Chiba, Chiba, Japan

34 CEICO, Institute of Physics, Czech Academy of Sciences, Prague, Czech Republic

${ }^{35}$ Department of Physics and Institute for the Early Universe, Ewha Womans University, Seodaaemun-gu, Seoul, Korea 